\title{
Silk Fibroin Nanoparticles for Drug Delivery: Effect of Bovine Serum Albumin and Magnetic Nanoparticles Addition on Drug Encapsulation and Release
}

\author{
Olga Gianak $^{1}$, Eleni Pavlidou ${ }^{2}$, Charalambos Sarafidis ${ }^{2}$, Vassilis Karageorgiou ${ }^{3}$ (1) \\ and Eleni Deliyanni ${ }^{4, *(1)}$ \\ 1 Division of Analytical Chemistry, Department of Chemistry, Aristotle University of Thessaloniki, \\ 54124 Thessaloniki, Greece; gianakolga@hotmail.com \\ 2 Department of Physics, Aristotle University of Thessaloniki, 54124 Thessaloniki, Greece; \\ elpavlid@auth.gr (E.P.); hsara@auth.gr (C.S.) \\ 3 Department of Food Technology, ATEI of Thessaloniki, P.O. BOX 141, 57400 Thessaloniki, Greece; \\ vkarageorgiou@food.teithe.gr \\ 4 Division of Chemical Technology, Department of Chemistry, Aristotle University of Thessaloniki, \\ 54124 Thessaloniki, Greece \\ * Correspondence: lenadj@chem.auth.gr; Tel.: +30-2310-997808
}

Received: 4 February 2018; Accepted: 9 April 2018; Published: 23 April 2018

\begin{abstract}
Silk fibroin nanoparticles were prepared in the present study based on phase separation between silk fibroin and polyvinyl alcohol. The drug encapsulation efficiency of the prepared nanoparticles was examined at a range of concentrations from $10 \mathrm{ppm}$ to $500 \mathrm{ppm}$ for pramipexole, curcumin, and propranolol hydrochloride. Silk fibroin nanoparticles encapsulated with propranolol presented the highest drug release profile. In order to improve the drug encapsulation efficiency and drug release performance, a modification of silk fibroin nanoparticles with bovine serum albumin and magnetic nanoparticles was tried. The modification was found to improve the drug encapsulation and release of the modified nanoparticles. Bovine-serum-modified nanoparticles presented the best improvement.
\end{abstract}

Keywords: silk fibroin; drug delivery; magnetic silk fibroin; bovine serum albumin

\section{Introduction}

Drug delivery aims to bring the compound with pharmaceutical activity to the exact point of need in the organism with the right concentrations in order to increase the efficiency of action [1]. The improvement of cellular uptake, the reduction of the side effects, the control of drug release, the enhancement of drug bioavailability, and the reduction of the drug degradation rate are the purposes of drug delivery systems [2]. Recently, nanoparticles are considered to be suitable for drug delivery due to their ability to act as modifiable platforms, their tunable size, and their high surface-to-volume ratio [2]. In addition, they can be used to deliver hydrophilic and hydrophobic drug molecules as well [3].

Silk fibroin, from the silk worm Bombyx mori, consists of hydrophobic and hydrophilic regions [2]. The hydrophobic domains, i.e., protein crystals and beta sheets, are dominated by repeats of alanine, glycine-alanine, and glycine-alanine-serine. Recently, silk fibroin, due to its excellent properties, such as biocompatibility, biodegradability, and low immunogenicity, has been extensively tested for a drug delivery application since silk materials exhibit high encapsulation efficiency and controllable drug release kinetics [4-6]. 
Bovine serum albumin (BSA) is a water-soluble protein with a well-defined structure. Positively or negatively charged drug molecules can be non-covalently bound to the charged amino acids of albumin. Silk fibroin contains hydrophobic amino acids that could create strong electrostatic interactions through the amino acids' carboxyl groups and the amino groups of bovine serum albumin. These interactions prevent leakage of hydrophobic drugs from bovine serum albumin; thereby, it is expected to improve drug encapsulation, delivery to affected body areas, and drug release rate [7]. Albumin was found to enable the localization of drug carriers in specific tissues (i.e., liver and heart tissues), regulating in this way biodistribution and drug release [8]. Besides this, it was shown that readily available and inexpensive serum albumin can overcome many drugs' shortcomings, such as insolubility, instability in biological environments, poor uptake into cells and tissues, sub-optimal selectivity for targets, and unwanted side effects [9].

Additionally, magnetic carriers and external magnetic fields that focalize on tumors have emerged as a hopeful strategy to enhance drug accumulation at tumor sites. Magnetic targeting has the advantage of not requiring complex chemical modification of targeting ligands on the surface of nanocarriers when compared with conventional tumor targeting. Exploiting a magnetic field as a driving force represents a noninvasive therapeutic approach [10,11].

In the present study, we report aqueous-based preparation methods for silk fibroin nanoparticles, based on phase separation between silk fibroin and polyvinyl alcohol (PVA), with a simple, inexpensive, and appropriate method for pharmaceutical and biomedical applications of drug delivery. Curcumin, pramipexole, and propranolol hydrochloride were chosen as model drugs in this study for their different molecular weights (MWs), surface chemistries, and disease targets. Curcumin, a natural compound with diphenolic groups, is extracted from the rhizome of turmeric. It has been used in clinical trials for cancer therapy $[12,13]$. Propranolol is a non-selective beta adrenergic blocking agent and has been used in the treatment of hypertension, angina pectoris, and many other cardiovascular disorders [14]. Pramipexole is a potent dopamine $\mathrm{D}_{2}$ agonist with a preference for $\mathrm{D}_{3}$ receptors and is approved worldwide for the treatment of Parkinson's disease symptoms [15].

Moreover, nanoparticles of silk fibroin were modified with bovine serum albumin for the enrichment of silk fibroin with the above-mentioned properties of albumin [7-9]. Silk fibroin-bovine serum albumin nanoparticles were prepared since bovine serum albumin has been found to be biocompatible, biodegradable, non-toxic, and non-immunogenic and has interesting results in drug encapsulation and release [16]. Finally, magnetic silk fibroin nanoparticles were also prepared and examined to identify the contribution of magnetic nanoparticles to the encapsulation efficiency and in vitro drug release of the model drugs.

\section{Materials and Methods}

\subsection{Materials}

Polyvinyl alcohol high molecular weight solid, (PVA 98-99 hydrolized) and curcumin (95\% total curcuminoid content) from Tumeric rhizome were purchased from A Johnson Company (New Brunswick, NJ, USA). Ethanol, Lithium Bromide 99\%, and Bovine Serum Albumin (BSA) (lyophilized powder, $66.000 \mathrm{kDa}$ ), were purchased from Sigma-Aldrich (St. Louis, MO, USA). Propranolol hydrochloride and Pramipexole were purchased from Fagron Hellas (Trikala, Greece). $\mathrm{FeCl}_{3} \bullet 6 \mathrm{H}_{2} \mathrm{O}, \mathrm{FeCl}_{2} \bullet 4 \mathrm{H}_{2} \mathrm{O}$, and $\mathrm{NH}_{4} \mathrm{OH}$ of analytical grade were purchased from Sigma-Aldrich.

\subsection{Methods}

\subsubsection{Preparation of Silk Fibroin Solution}

Silk fibroin aqueous solution was prepared according to described protocols [17]. Briefly, $5 \mathrm{~g}$ cocoons of Bombyx mori were boiled in $2 \mathrm{~L}$ of an aqueous solution of sodium carbonate $(0.02 \mathrm{M})$ for $30 \mathrm{~min}$ and the degummed silk fibroin was thoroughly rinsed with deionized water, air dried 
overnight, and then dissolved in a $9.3 \mathrm{M} \mathrm{LiBr}$ solution at $60{ }^{\circ} \mathrm{C}$. The solution was dialyzed against deionized water using Slide-a-Lyzer dialysis cassettes (MWCO 3.500, Pierce, Thermo-Fischer Scientific, Waltham, MA, USA) for 2 days for salt removal. In order to clean the solution from impurities, silk aggregates, and debris of cocoons, the silk fibroin solution was centrifuged twice at $9000 \mathrm{rpm}$ for $30 \mathrm{~min}$. The prepared silk fibroin solution was stored at $4{ }^{\circ} \mathrm{C}$ for further use.

\subsubsection{Preparation of Silk Fibroin (SF) Nanoparticles}

Silk fibroin particles were prepared according to the following method [18]: $5 \mathrm{~mL}$ of silk fibroin solution was mixed with $2 \mathrm{~mL}$ ethanol with a sample pipette and then vortexed for $10 \mathrm{~s}$. Subsequently, $50 \mathrm{~mL}$ of $5 \%$ PVA solution were added to the fibroin/ethanol mixture and vortexed for $10 \mathrm{~s}$. The fibroin/ethanol/PVA solution mixture was refrigerated for $24 \mathrm{~h}$ and centrifuged. In order to remove PVA and ethanol from the nanoparticles, the mixture was washed with deionized water three times and centrifuged after each wash [18].

\subsubsection{Preparation of Silk Fibroin-Bovine Serum Albumin (SF-BSA) Nanoparticles}

For the preparation of the Silk Fibroin-Bovine Serum Albumin nanoparticles (SF-BSA-NPs), $2.5 \mathrm{~mL}$ of the silk fibroin solution were mixed with $2.5 \mathrm{~mL}$ of $2 \%$ BSA solution and then the mixture was stirred for 15-20 min. The SF-BSA-NPs were prepared following the method used to prepare the silk fibroin nanoparticles.

\subsubsection{Synthesis of $\mathrm{Fe}_{3} \mathrm{O}_{4}$ Nanoparticles}

For the preparation of the magnetic silk fibroin nanoparticles (SFm-NPs), the magnetic material used was magnetite nanoparticles $\left(\mathrm{Fe}_{3} \mathrm{O}_{4}\right)$ prepared in the laboratory. The $\mathrm{Fe}_{3} \mathrm{O}_{4}$ nanoparticles were prepared according to the modified Massart method [19] via the co-precipitation of $\mathrm{FeCl}_{3} \bullet 6 \mathrm{H}_{2} \mathrm{O}$ and $\mathrm{FeCl}_{2} \bullet 4 \mathrm{H}_{2} \mathrm{O}$ [18]; briefly, $11.2 \mathrm{mmol} \mathrm{FeCl}{ }_{3} \bullet 6 \mathrm{H}_{2} \mathrm{O}$ and $5.6 \mathrm{mmol} \mathrm{FeCl}_{2} \bullet 4 \mathrm{H}_{2} \mathrm{O}$ were dissolved in $150 \mathrm{~mL}$ of deionized water, heated to $60^{\circ} \mathrm{C}$ under agitation in an inert atmosphere, and then aqueous ammonia solution was added dropwise until the solution's $\mathrm{pH}$ became 10. The precipitate that was formed was magnetically collected, washed with deionized water and ethanol, and freeze-dried.

\subsubsection{Preparation of Silk Fibroin- $\mathrm{Fe}_{3} \mathrm{O}_{4}(\mathrm{SFm})$ Nanoparticles}

For the preparation of the magnetic silk fibroin nanoparticles (SFm-NPs), $0.1 \mathrm{~g} \mathrm{Fe}_{3} \mathrm{O}_{4}$ was added to $5 \mathrm{~mL}$ of silk fibroin solution followed by the preparation of nanoparticles as above described.

\subsubsection{Preparation of Drug-Encapsulated Silk Fibroin Nanoparticles}

Curcumin $(\mathrm{MW}=368.39 \mathrm{~g} / \mathrm{mol})$, propranolol hydrochloride $(\mathrm{MW}=259.34 \mathrm{~g} / \mathrm{mol})$, and pramipexole ( $\mathrm{MW}=211.32 \mathrm{~g} / \mathrm{mol}$ ) were the model drugs tested for the encapsulation in the silk fibroin nanoparticles; the chemical formulas of the drugs are presented in Table 1.

Amounts of the pramipexole and propranolol were dissolved in deionized water and mixed with $5 \mathrm{~mL}$ of the silk fibroin solution; curcumin was dissolved in ethanol and then mixed with $5 \mathrm{~mL}$ of the silk fibroin solution. Different amounts of the drugs were estimated for the preparation of solutions with concentrations of 10, 35, 50, 100, 200, and 500 ppm for each drug. Finally, the drug-encapsulated silk fibroin nanoparticles were prepared as previously described.

The encapsulation efficiency in the silk fibroin nanoparticles was determined by measuring the UV-vis absorbance (HITACHI U-2000) of the supernatant after the centrifugation that follows the formation of silk fibroin nanoparticles at 430, 265, and $235 \mathrm{~nm}$ for curcumin, pramipexole, and propranolol, respectively. Standard calibration curves of model drugs were used for drug quantification $\left(r^{2} \geq 0.999\right)$. All experiments were performed in triplicate and the mean value is presented. The difference between the total amount of drug used in the experiment and the amount 
that remained in the supernatants was expressed as encapsulation efficiency (EE) in silk fibroin nanoparticles [20] and determined by the following equation:

$$
\text { Encapsulation Efficiency }=\frac{\text { amount of drug that remained in the particles }}{\text { total amount of drug used }} \times 100
$$

Table 1. Model drugs encapsulated in silk fibroin nanoparticles.

propranolol hydrochloride

MW = molecular weight.

\subsubsection{Silk Fibroin Nanoparticles Characterization}

For the identification of the crystalline phase of silk fibroin nanoparticles, $X$-ray powder diffraction (XRD) patterns were recorded on a Philips PW 1820 diffractometer (Amsterdam, The Netherlands) with $\mathrm{Cu} \mathrm{K} \alpha$ radiation from $20^{\circ}$ to $60^{\circ}$. The morphology of silk fibroin nanoparticles as well as that of drug-encapsulated silk fibroin nanoparticles was imaged using a JEOL JMS-840A scanning electron microscope (JEOL, Tokyo, Japan). The control samples of silk fibroin nanoparticles suspensions in water, the samples of SF-BSA-NPs and SFm-NPs, and their respective drug-encapsulated nanoparticles suspensions in water were lyophilized and the powder obtained was subjected to Fourier Transform Infrared measurement using a Perkin-Elmer FTIR spectrophotometer (model Spectrum 1000, Rodgau, Germany). Nanoparticle size, size distribution, and surface charges of the nanoparticles, and those of their respective drug-encapsulated nanoparticles, were determined by Dynamic Light Scattering (DLS) with a NanoBrook ZetaPALS Brookhaven Instruments (Holtsville, NY, USA) equipped with a diode laser (wavelength, $\lambda=532 \mathrm{~nm}$ ). Hysteresis loops for the SF-magnetite nanoparticles were recorded with an Oxford 1.2 H/CF/HT vibrating sample magnetometer (VSM).

\subsubsection{In Vitro Drug Release}

The amount of drug released in vitro from the drug-encapsulated silk fibroin nanoparticles, at specific time intervals, was calculated as follows: $4 \mathrm{~mL}$ of the drug-encapsulated nanoparticles were added to $20 \mathrm{~mL}$ of phosphate buffer saline (PBS) at pH 7.4 and shaken at a rate of $120 \mathrm{rpm}$ at $37^{\circ} \mathrm{C}$. At specified time intervals, $2 \mathrm{~mL}$ were sampled and centrifuged at $9000 \mathrm{rpm}$ for $10 \mathrm{~min}$. The concentration of the drug in the supernatant was measured by UV-vis spectrometry at 430, 265, and $235 \mathrm{~nm}$ for curcumin, pramipexole, and propranolol, respectively. The percentage release was determined as the ratio of the measured amount of the drug released at different time intervals to the initial amount of drug encapsulated in the nanoparticles [12]. All experiments were performed in triplicate and the mean value is presented. 


\section{Results and Discussion}

Weighting the residual solid of a certain known volume of silk solution after drying at $60{ }^{\circ} \mathrm{C}$, the concentration of the silk fibroin aqueous solution prepared was found to be approximately $4.6 \%(w / v)$.

\subsection{XRD Characterization, Morphology, and FTIR Characterization of the Silk Fibroin Nanoparticles}

XRD peaks of silk fibroin are associated with its crystalline structure; Figure 1 presents the XRD results of the silk fibroin nanoparticles prepared in the current study. The $2 \theta$ diffraction peak presented in the XRD pattern of the silk fibroin nanoparticles at $2 \theta=19.2^{\circ}$ could be attributed to the silk II crystal structure of fibroin, indicating an increased crystallization degree of silk fibroin in the nanoparticles [21,22].

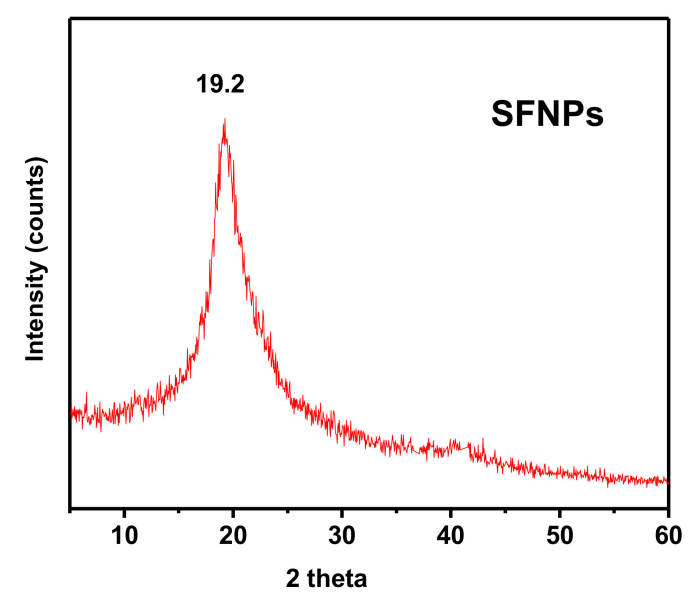

Figure 1. XRD patterns of silk fibroin nanoparticles (SFNPs).

The morphology of silk fibroin nanoparticles was illustrated by SEM images and is presented in Figure 2a. The nanoparticles observed presented a spherical and/or cylindrical shape with an average mean diameter of about $290 \mathrm{~nm}$. Silk fibroin nanoparticles previously prepared with the same method by Shi et al. [18] presented an average diameter of about $686.5 \mathrm{~nm}$ as estimated by SEM image. This difference in size could be attributed to the lower concentration ( $4 \%)$ of silk fibroin solution that was used in this study to prepare the silk fibroin nanoparticles compared to the study by Shi et al. (6\%). According to Zhao et al., the increase of the starting silk fibroin solution concentration has an effect on the particle size increase [23].
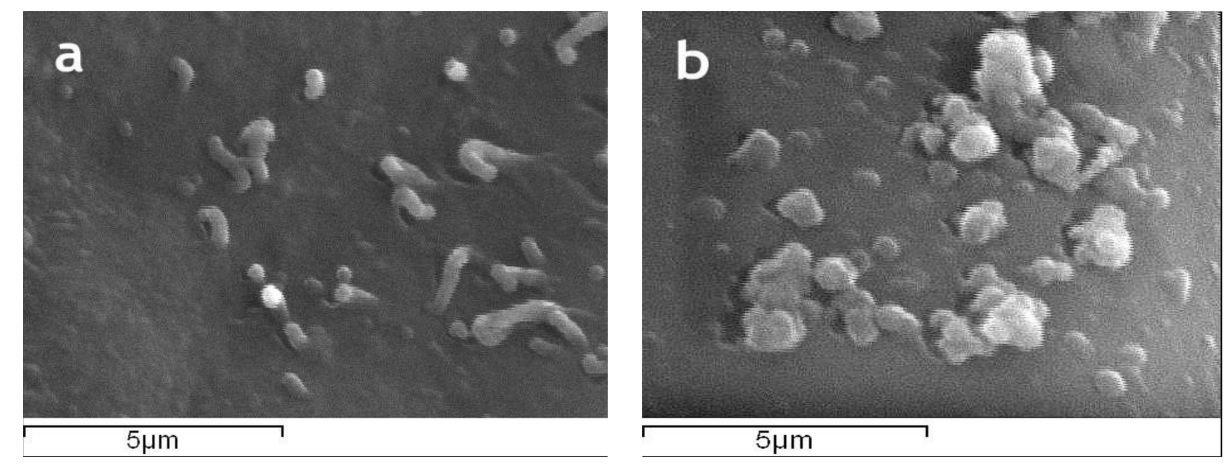

Figure 2. Cont. 

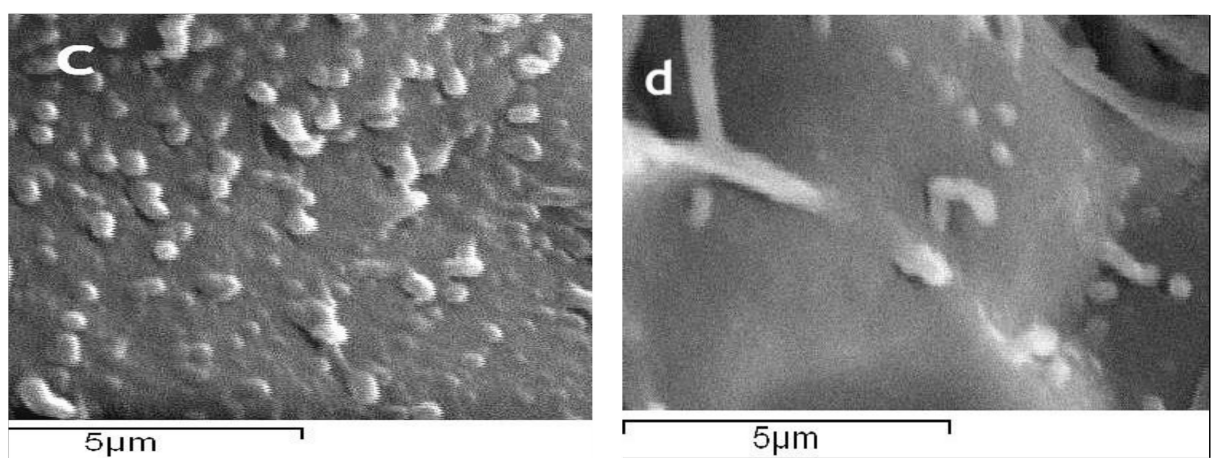

Figure 2. SEM images of SF nanoparticles (a); SF-propanolol NPs (b); SF-pramipexole NPs (c); and SF-curcumin NPs (d).

In Figure 3, the FTIR spectrum of the prepared silk fibroin nanoparticles is presented. The characteristic peak at $1632 \mathrm{~cm}^{-1}$ attributed to an amide I $\beta$-sheet and the peak at $1260 \mathrm{~cm}^{-1}$ attributed to amide III are due to random coil. So, when the nanoparticles of silk fibroin were formed both the silk I crystal structure and the silk II crystal structure of silk fibroin were observed [24,25]. The assignments of the FTIR bands of silk fibroin nanoparticles are presented in detail in Table 2.

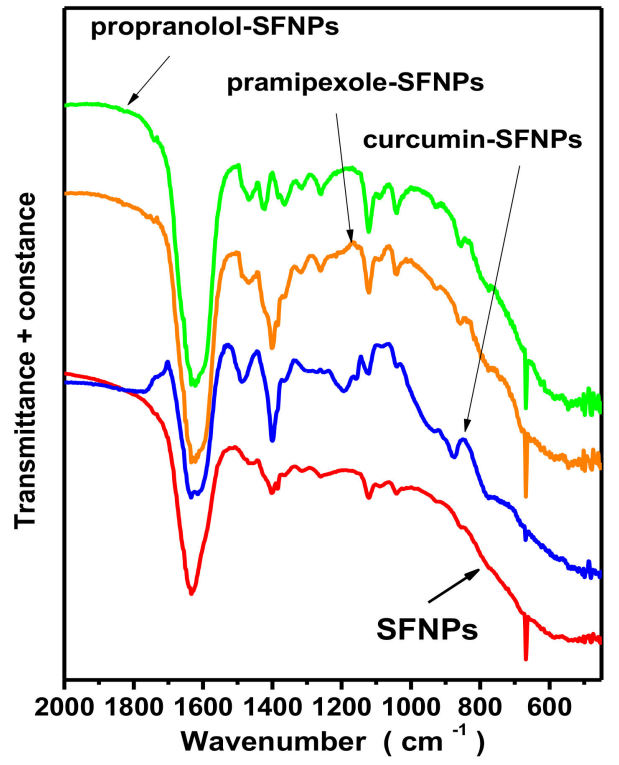

Figure 3. FTIR spectra of silk fibroin nanoparticles and drug-encapsulated silk fibroin nanoparticles. 
Table 2. Assignments of the FTIR bands of silk fibroin nanoparticles and drug-encapsulated silk fibroin nanoparticles.

\begin{tabular}{|c|c|c|c|c|c|c|c|}
\hline $\mathrm{cm}^{-1}$ & SF Nanoparticles & $\mathrm{cm}^{-1}$ & SF-Pramipexole NPs & $\mathrm{cm}^{-1}$ & SF-Propranolol NPs & $\mathrm{cm}^{-1}$ & SF-Curcumin NPs \\
\hline 1632 & $\begin{array}{l}\text { Amide I } \\
\beta \text {-sheets }\end{array}$ & 1627 & $\begin{array}{l}\text { Amide I } \\
\beta \text {-sheets }\end{array}$ & 1627 & $\begin{array}{l}\text { Amide I } \\
\beta \text {-sheets }\end{array}$ & 1628 & $\begin{array}{l}\text { Amide I } \\
\beta \text {-sheets }\end{array}$ \\
\hline 1465 & $\mathrm{CH}_{3}$ bending & 1469 & $\mathrm{CH}_{3}$ bending & 1465 & $\mathrm{CH}_{3}$ bending & 1488 & curcumin \\
\hline 1401 & $\mathrm{CH}_{3}$ bending & & & 1422 & $\mathrm{CH}_{3}$ bending & 1399 & $\mathrm{CH}_{3}$ bending \\
\hline 1354 & $\mathrm{CH}_{2}$ bending $(\mathrm{AG})_{n}$ & & & 1367 & $\mathrm{CH}_{2}$ bending $(\mathrm{AG})_{n}$ & & \\
\hline 1311 & OCO- stretching & 1315 & OCO- stretching & 1319 & OCO- stretching & 1303 & $\begin{array}{c}\text { Amide III } \\
\beta \text {-turn }\end{array}$ \\
\hline \multirow[t]{2}{*}{1260} & $\begin{array}{c}\text { Amide III } \\
\text { a-helices }\end{array}$ & 1260 & $\begin{array}{c}\text { Amide III } \\
\text { a-helices }\end{array}$ & 1260 & $\begin{array}{l}\text { Amide III } \\
\text { a-helices }\end{array}$ & & \\
\hline & & & & & & 1192 & curcumin \\
\hline 1123 & $\begin{array}{c}\mathrm{CH}_{2} \mathrm{OH} \\
\text { polyphenols }\end{array}$ & 1118 & $\begin{array}{c}\mathrm{CH}_{2} \mathrm{OH} \\
\text { polyphenols }\end{array}$ & 1118 & $\begin{array}{c}\mathrm{CH}_{2} \mathrm{OH} \\
\text { polyphenols }\end{array}$ & & \\
\hline 1041 & $\begin{array}{c}\text { C-O stretching } \\
\mathrm{C}-\mathrm{OH} \text { stretching } \\
\text { Ser }\end{array}$ & 1041 & $\begin{array}{c}\mathrm{C}-\mathrm{O} \text { stretching } \\
\mathrm{C}-\mathrm{OH} \text { stretching } \\
\text { Ser }\end{array}$ & 1041 & $\begin{array}{l}\mathrm{C}-\mathrm{O} \text { stretching } \\
\mathrm{C}-\mathrm{OH} \text { stretching } \\
\text { Ser }\end{array}$ & 1036 & $\begin{array}{l}\mathrm{C}-\mathrm{O} \text { stretching } \\
\mathrm{C}-\mathrm{OH} \text { stretching } \\
\text { Ser }\end{array}$ \\
\hline \multirow[t]{2}{*}{926} & $\begin{array}{c}(\mathrm{A})_{n} \\
\beta \text {-sheets }\end{array}$ & 926 & $\begin{array}{c}(\mathrm{A})_{n} \\
\beta \text {-sheets }\end{array}$ & 926 & $\begin{array}{c}(\mathrm{A})_{n} \\
\beta \text {-sheets }\end{array}$ & & \\
\hline & & & & & & 873 & curcumin \\
\hline
\end{tabular}

$(\mathrm{A})_{n}$, polyalanine; $(\mathrm{AG})_{n}$, polyalanine glycine [26].

\subsection{Encapsulation Efficiency}

The encapsulation efficiency (EE) of the silk fibroin nanoparticles for the three model drugs was studied at a range of concentrations from 10 to $500 \mathrm{ppm}$. For the curcumin-encapsulated silk fibroin nanoparticles the highest EE was found to be $98 \pm 2 \%$ and was achieved at $500 \mathrm{ppm}$, for propranolol the highest EE was $69 \pm 3 \%$ at 50 ppm and for pramipexole the highest EE was $68 \pm 2 \%$ at $35 \mathrm{ppm}$ (data not shown).

\subsection{Size, $\zeta$-Potential, Morphology, and FTIR Characterization of Silk Fibroin Nanoparticles} and Drug-Encapsulated Silk Fibroin Nanoparticles

The size of silk fibroin nanoparticles measured by DLS is presented in Table 3. As seen in the Table, the average diameter of the silk fibroin nanoparticles was found to be $301 \pm 11 \mathrm{~nm}$, which is consistent with the size estimated by SEM; the average size was found to increase after the encapsulation of the model drugs (curcumin, pramipexole, and propranolol) in the silk fibroin nanoparticles. The polydispersity index, ranging from 0.10 to 0.25 , revealed a uniform particle distribution.

Table 3. Diameter of silk fibroin nanoparticles measured by dynamic light scattering.

\begin{tabular}{ccc}
\hline & Diameter \pm SD $(\mathbf{n m})(\boldsymbol{n}=\mathbf{5})$ & Polydispersity Index \\
\hline SFNPs & $301 \pm 11$ & 0.24 \\
SF-curcumin NPs & $354 \pm 13$ & 0.25 \\
SF-pramipexole NPs & $469 \pm 14$ & 0.10 \\
SF-propranolol NPs & $501 \pm 7$ & 0.20 \\
\hline
\end{tabular}

The surface charge of the silk fibroin solution, of the drug solutions, of the silk fibroin nanoparticles, and of the silk fibroin nanoparticles encapsulated with the model drugs was determined by $\zeta$-potential and the results are presented in Table 4 . For all samples, the $\zeta$-potential was negative; it was observed that the $\zeta$-potential of propranolol-encapsulated silk fibroin nanoparticles (SF-propranolol NPs) was higher than that of curcumin (SF-curcumin NPs) and pramipexole-encapsulated silk fibroin nanoparticles (SF-pramipexole NPs), leading to the conclusion 
that propranolol was encapsulated in the center of the silk fibroin nanoparticles while curcumin and pramipexol were adsorbed on the surface of the nanoparticles.

Table 4. $\zeta$-potential of model drug solutions, silk fibroin nanoparticles, and drug-encapsulated silk fibroin nanoparticles.

\begin{tabular}{cc}
\hline Sample & $\zeta$-Potential $\pm \mathrm{SD}(\mathrm{mV})(n=3)$ \\
\hline Fibroin solution 4.1\% & $-4.3 \pm 0.1$ \\
Curcumin solution 500 ppm & $-7 \pm 2$ \\
Pramipexole solution 35 ppm & $-0.06 \pm 0.02$ \\
Propranolol solution 50 ppm & $-2.1 \pm 0.3$ \\
Silk fibroin nanoparticles & $-16.40 \pm 0.02$ \\
SF-curcumin NPs & $-5.5 \pm 0.2$ \\
SF-pramipexole NPs & $-6 \pm 2$ \\
SF-propranolol NPs & $-14.9 \pm 0.3$ \\
\hline
\end{tabular}

The morphology of drug-encapsulated silk nanoparticles, and that of the silk nanoparticles for the sake of comparison, was illustrated by SEM images (Figure 2). The nanoparticles observed presented a spherical and/or cylindrical shape with an average mean diameter of $290 \mathrm{~nm}$. As expected, the size of silk fibroin nanoparticles increased after drug encapsulation. The mean diameter of the drug-encapsulated silk fibroin nanoparticles, as estimated from the SEM images, was 490, 392, and $294 \mathrm{~nm}$ for propranolol-, pramipexole-, and curcumin-encapsulated silk fibroin nanoparticles, respectively. It is worth noting that these results for nanoparticle sizes are relatively smaller than those measured with DLS; this can be attributed to the dry state of the nanoparticles when measured with SEM.

In Figure 3, the FTIR spectra of silk fibroin nanoparticles and of drug-encapsulated silk fibroin nanoparticles are presented. In the spectra of the curcumin-encapsulated silk fibroin nanoparticles, it was observed that certain characteristic peaks of the original curcumin were either not evident or exhibited a slight shift or alteration, which demonstrated that certain structural changes and chemical reactions may have occurred between the curcumin and the silk fibroin [12,27]. Curcumin showed its characteristic group absorption peaks in sharp absorption bands at $1605 \mathrm{~cm}^{-1}, 1502 \mathrm{~cm}^{-1}(-\mathrm{C}=\mathrm{O}$ and $-\mathrm{C}-\mathrm{C}$ vibrations), $1435 \mathrm{~cm}^{-1}$ (an olefinic $-\mathrm{C}-\mathrm{H}$ bending vibration), $1285 \mathrm{~cm}^{-1}$ (an aromatic $-\mathrm{C}-\mathrm{O}$ stretching vibration), and $833 \mathrm{~cm}^{-1}$ (a C-H bond of alkene group) [12,27]. In addition, propranolol exhibited characteristic peaks in sharp absorption bands at $1680-1620 \mathrm{~cm}^{-1}$ (C=C stretching) and 1260-1000 $\mathrm{cm}^{-1}$ (C-O stretching) [28]. On the contrary, the FTIR spectrum of pramipexole-encapsulated fibroin nanoparticles presented no difference compared to pure fibroin nanoparticles; the characteristic peaks of pramipexol were not evident, leading to the conclusion that after encapsulation with pramipexole the chemical bonding remained unchanged [29]. The assignments of the FTIR bands of silk fibroin nanoparticles as well as those of the drug-encapsulated silk fibroin nanoparticles are presented in detail in Table 4.

\subsection{In Vitro Drug Release}

The drug release profile, presented in Figure 4, indicated that the encapsulated curcumin and pramipexole silk fibroin nanoparticles had a short and low-level release percentage, about $1.2 \%$ and $0.25 \%$, respectively (presented also in the insets of the Figure 4 ) while the propranolol-encapsulated silk fibroin nanoparticles exhibited a release of about $65 \%$ within 6 days. This could be due to the lack of interactions of propranolol with the silk fibroin as well as to the hydrophilic properties of propranolol. Curcumin, as a hydrophobic drug, was possibly attached to silk fibroin via hydrophobic interactions and $\pi-\pi$ stacking, and presented a reduced release rate compared with that of the hydrophilic drug propranolol $[4,12,30]$. Pramipexole was expected to be attached to silk fibroin through strong electrostatic interactions attributed to the $-\mathrm{NH}_{2}$ groups, which could have resulted in the decreased release rate. 


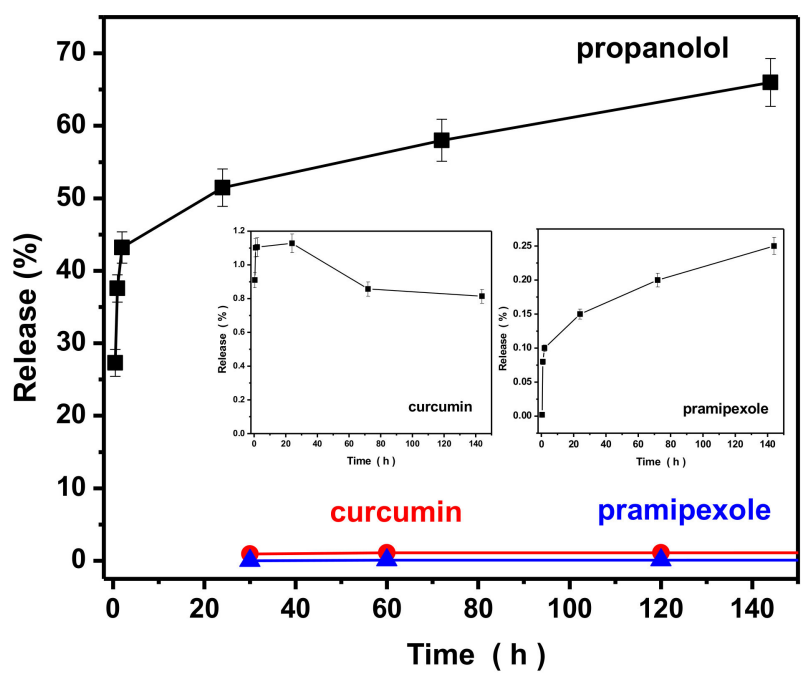

Figure 4. Drug release rate of model drugs from nanoparticles of silk fibroin.

\subsection{Modification of SF with Bovine Serum Albumin and Magnetic Nanoparticles}

In order to enhance the properties of silk fibroin nanoparticles, SFm-NPs and silk fibroin-bovine serum albumin nanoparticles (SF-BSA-NPs) were prepared via the introduction of magnetic nanoparticles $\left(\mathrm{Fe}_{3} \mathrm{O}_{4}\right)$ and BSA during the silk fibroin nanoparticle formulation, and the drug encapsulation and release efficiency of these silk fibroin nanoparticles were examined in detail. Since propranolol presented the highest encapsulation and release performance, it was used as the model drug for the performance of the two modified types of silk fibroin nanoparticles.

\subsubsection{Characterization of Modified Nanoparticles}

DLS measurements for silk fibroin-bovine serum albumin nanoparticles and magnetic silk fibroin nanoparticles are presented in Table 5. Both samples indicated a small polydispersity index of 0.30 and 0.36, respectively, indicating a uniform diameter distribution of nanoparticles.

Table 5. Diameter of magnetic and fibroin-albumin nanoparticles measured by Dynamic Light Scattering.

\begin{tabular}{ccc}
\hline Sample & Diameter \pm SD $(\mathbf{n m})(\boldsymbol{n}=\mathbf{5})$ & Polydispersity Index \\
\hline SFNPs & $301 \pm 11$ & 0.24 \\
SFm-NPs & $337 \pm 14$ & 0.30 \\
SF-BSA-NPs & $268 \pm 4$ & 0.36 \\
\hline
\end{tabular}

SFm-NPs = magnetic silk fibroin nanoparticles; SF-BSA-NPs = silk fibroin-bovine serum albumin nanoparticles.

The encapsulation efficiency of magnetic silk fibroin and silk fibroin-bovine serum albumin nanoparticles is presented in Table 6. From the Table, it can be seen that the modification of silk fibroin with magnetic nanoparticles and bovine serum albumin increased the drug encapsulation compared to the pure silk fibroin nanoparticles. Propranolol is a hydrophilic drug and the presence of bovine serum albumin makes the silk fibroin-bovine serum albumin nanoparticles more hydrophilic; for this reason the encapsulation efficiency was increased. Also, the increased encapsulation efficiency of magnetic silk fibroin loaded with propranolol may be attributed to the adsorption of propranolol on $\mathrm{Fe}_{3} \mathrm{O}_{4}$. 
Table 6. Encapsulation Efficiency (EE) of magnetic silk fibroin and silk fibroin-bovine serum albumin nanoparticles encapsulated with propranolol.

\begin{tabular}{cccc}
\hline C (ppm) & $\begin{array}{c}\text { EE } \pm \text { SD (\%) } \\
\text { Propranolol SFNPs } \\
(\boldsymbol{n}=3)\end{array}$ & $\begin{array}{c}\text { EE } \pm \text { SD (\%) } \\
\text { Propranolol SFm-NPs } \\
(\boldsymbol{n}=\mathbf{3})\end{array}$ & $\begin{array}{c}\text { EE } \pm \text { SD (\%) } \\
\text { Propranolol SF-BSA-NPs } \\
(\boldsymbol{n}=\mathbf{3})\end{array}$ \\
\hline 50 & $70 \pm 2$ & $96 \pm 4$ & $97 \pm 3$ \\
\hline
\end{tabular}

The surface morphology of magnetic silk fibroin particles and the silk fibroin-bovine serum albumin nanoparticles as well as their encapsulated-with-propranolol counterparts, illustrated by SEM images, is presented in Figure 5. The magnetic silk fibroin (SFm) and silk fibroin-bovine serum albumin nanoparticles (SF-BSA) exhibited a spherical shape with a diameter of about $288 \mathrm{~nm}$, while the propranolol-encapsulated silk fibroin-bovine serum albumin nanoparticles (SF-BSA + propranolol) and propranolol-encapsulated magnetic silk fibroin nanoparticles (SFm + propranolol) exhibited a particle size of about $384 \mathrm{~nm}$ and $490 \mathrm{~nm}$, respectively (Figure 5).
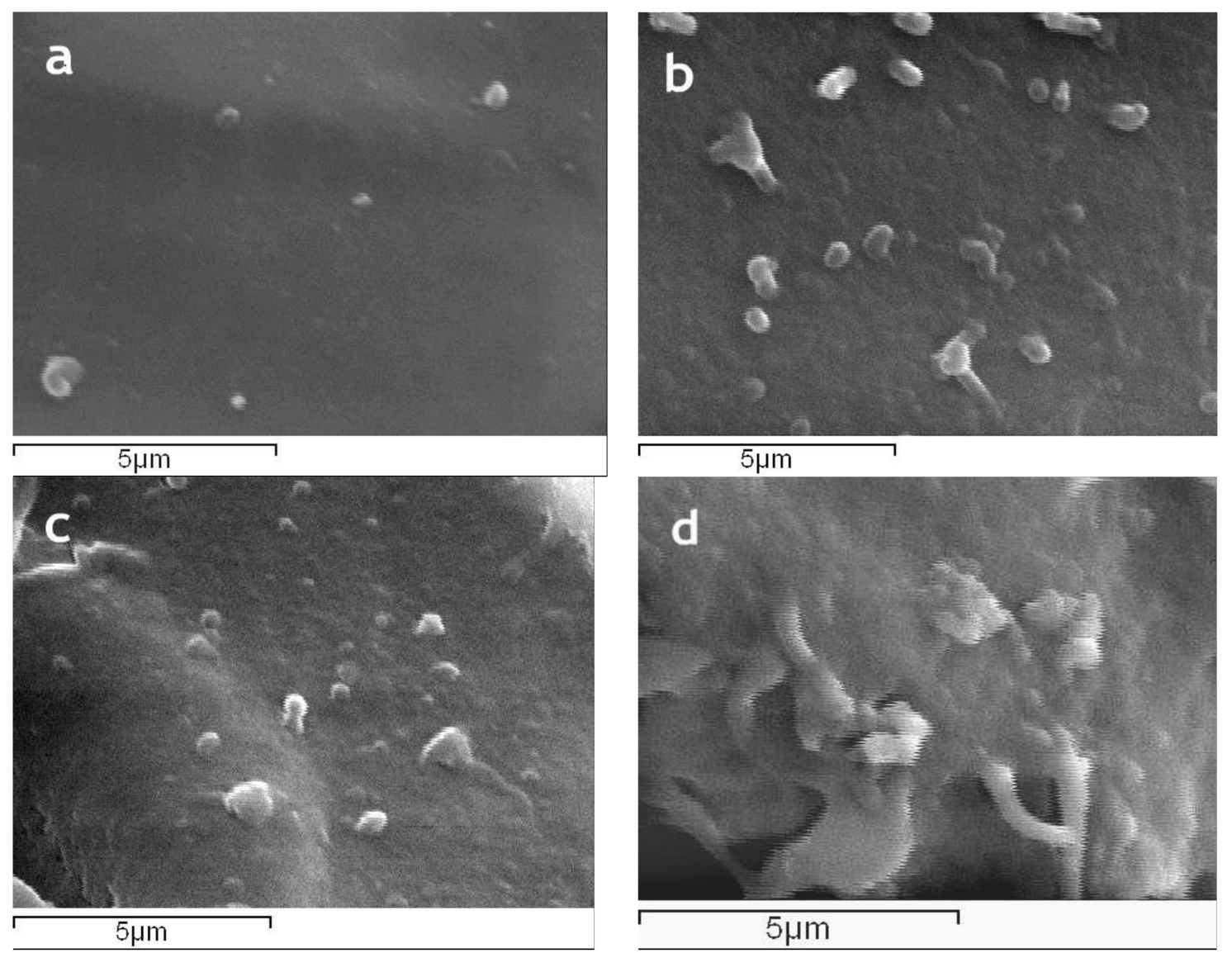

Figure 5. SEM images of SFm nanoparticles (a); SFm + propranolol NPs (b); SF-BSA-NPs (c); and SF-BSA + propranolol NPs (d).

The $2 \theta$ diffraction peak presented in the XRD pattern of the magnetic silk fibroin nanoparticles (Figure 6a), that appeared at $2 \theta=19.2^{\circ}$, could be attributed to silk I, a random coil of silk fibroin, while for the encapsulated-with-propranolol magnetic silk fibroin nanoparticles, the characteristic peak at $2 \theta=20.7^{\circ}$ could be attributed to silk II, a $\beta$-sheet of fibroin. In addition, diffraction peaks corresponding to the (3 11 ), (4 00 ), and (4 40 ) planes of the cubic crystal structure (fcc) of $\mathrm{Fe}_{3} \mathrm{O}_{4}$, show the formation of magnetic silk fibroin nanoparticles [19]. The aforementioned XRD results are in agreement with the FTIR results. 
Prior to the measurements, the instrument was calibrated against a NIST-certified Ni standard. Each sample had approximately the same dimensions and an additional calibration procedure was performed in order to minimize shape effects. All measurements were performed in a controlled room temperature of $22{ }^{\circ} \mathrm{C}$ and consisted of a gradual increase of the applied external magnetic field up to about $+1.9 \mathrm{~T}\left(\mu_{0} \mathrm{H}\right)$ then down to $-1.9 \mathrm{~T}$ and again up to $+1.9 \mathrm{~T}$ while measuring the magnetic moment of the sample. The magnetic properties are attributed to the $\mathrm{Fe}_{3} \mathrm{O}_{4}$ particles and the overall appearance of the loop is typical for nanoparticles of that composition and size. All measurements have exactly the same appearance, i.e., initial slope, curvature, and saturation plateau, indicating that there are no significant differences in the $\mathrm{Fe}_{3} \mathrm{O}_{4}$ particles between the samples and that the procedure for the preparation of the magnetic silk fibroin nanoparticles (SFm-NPs) does not affect significantly the magnetic properties of the $\mathrm{Fe}_{3} \mathrm{O}_{4}$ particles. Remanence, $\mathrm{Mr}$, the Mass Magnetization which corresponds to a zero field after the first application of the maximum external field equals to $5-7 \%$ of the saturation magnetization and coercivity, $\mu_{0} \mathrm{H}_{c}$, the reverse external field which zeroes the magnetic moment of the sample is about $0.01 \mathrm{~T}$. Both the remanence and the coercivity are very low in all cases as expected for a soft ferromagnetic material. Saturation magnetization, the maximum Mass Magnetization which could be observed, was obtained for the sample via extrapolation from data of Figure $6 \mathrm{~b}, \mathrm{c}$ and was found to be $\sigma_{\mathrm{s}}=27.0(8 \mathrm{~b})$ and $27.9(8 \mathrm{c}) \mathrm{emu} / \mathrm{g}$. These results correspond to about $50 \%$ wt. $\mathrm{Fe}_{3} \mathrm{O}_{4}$ content, since the saturation magnetization depends on the $\mathrm{Fe}_{3} \mathrm{O}_{4}$ content which is diluted in the silk fibroin, confirmed that $\mathrm{Fe}_{3} \mathrm{O}_{4}$ particles are successfully incorporated into the silk fibroin and that their magnetic properties are preserved after drug encapsulation.
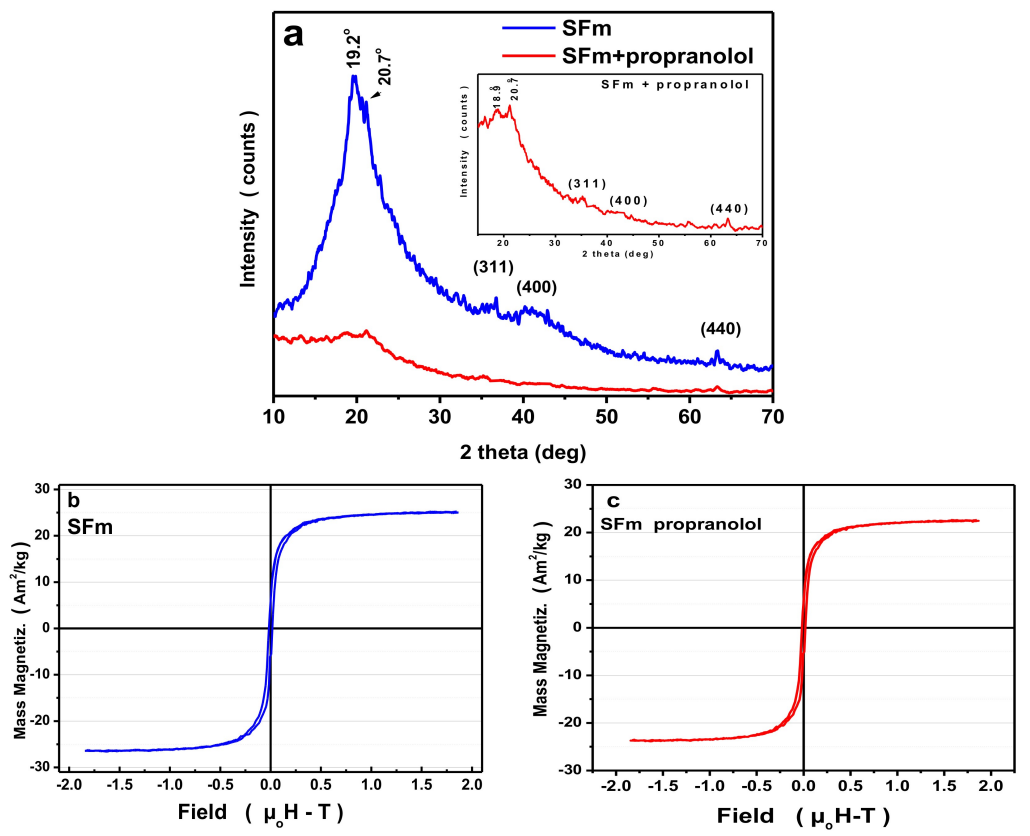

Figure 6. (a) XRD patterns of the magnetic silk fibroin nanoparticles and the propranolol-encapsulated magnetic silk fibroin nanoparticles; (b) hysteresis loop of the magnetic silk fibroin nanoparticles; and (c) hysteresis loop of the propranolol-encapsulated magnetic silk fibroin nanoparticles.

FTIR results of silk fibroin-bovine serum albumin nanoparticles and propranolol-encapsulated silk fibroin-bovine serum albumin nanoparticles are presented in Figure 7a,b, respectively, and the characteristic peaks are presented in Table 7. The bands at $1645 \mathrm{~cm}^{-1}$ and $1491 \mathrm{~cm}^{-1}$ are due to the BSA modification and can be attributed to amide I ( $\mathrm{C}=\mathrm{O}$ stretching) and amide II ( $\mathrm{C}-\mathrm{N}$ stretching and $\mathrm{N}-\mathrm{H}$ bending) vibrations of BSA, respectively (Table 7). The bands of BSA at $1392 \mathrm{~cm}^{-1}\left(-\mathrm{CH}_{2}\right.$ bending) and $\sim 1260 \mathrm{~cm}^{-1}$ (amide III, C-N stretching, and N-H bending) may be overlapped by the bands 
attributed to silk fibroin [7]. A slight shift in the spectra for the propranolol-loaded nanoparticles could be due to drug binding.
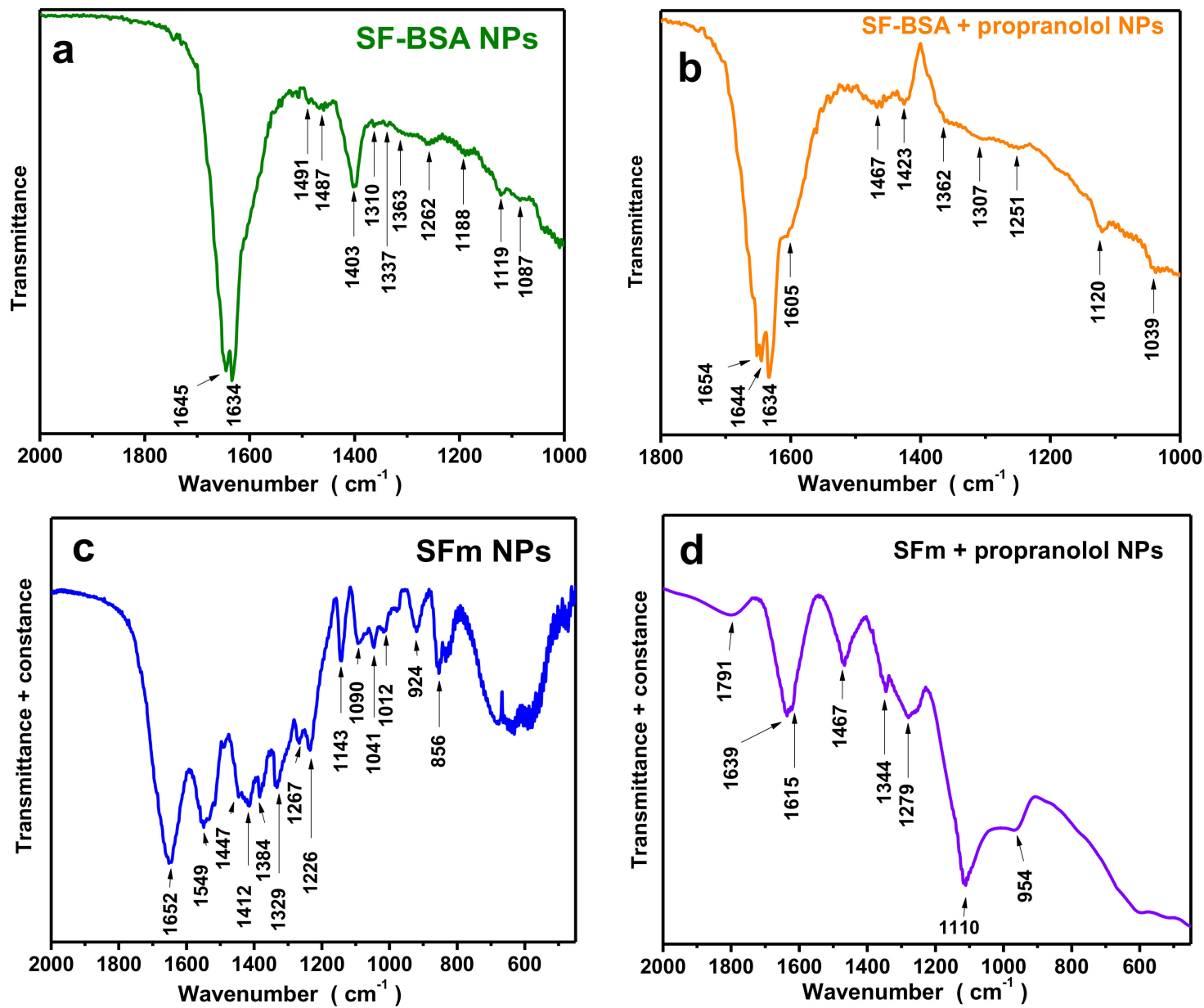

Figure 7. FTIR spectra of: (a) silk fibroin-bovine serum albumin nanoparticles; (b) propranolol-encapsulated silk fibroin-bovine serum albumin nanoparticles; (c) magnetic silk nanoparticles; and (d) propranolol-encapsulated magnetic silk fibroin nanoparticles.

In Figure $7 \mathrm{c}, \mathrm{d}$, the FTIR results of fibroin magnetic nanoparticles (SFm) and propranolol-encapsulated fibroin magnetic nanoparticles (SFm + propranolol) are presented, while the characteristic peaks are also presented in detail in Table 7. The spectra presented characteristic bands at 471 and $588 \mathrm{~cm}^{-1}$ and 506 and $595 \mathrm{~cm}^{-1}$, respectively, attributed to $\mathrm{Fe}-\mathrm{O}$ bonds [4], which confirmed the successful preparation of magnetic silk fibroin nanoparticles (Figure 7c). From the spectra, the existence of both $\beta$-sheets and a-helices for magnetic fibroin nanoparticles could be observed, while for the propranolol-encapsulated silk fibroin magnetic nanoparticles $\beta$-sheets could be also observed. 
Table 7. Assignment of the main bands present in SF-BSA NPs, SF-BSA + propranolol NPs, and SFm and SFm + propranolol NPs.

\begin{tabular}{|c|c|c|c|c|c|c|c|}
\hline $\mathrm{cm}^{-1}$ & SF-BSA NPs & $\mathrm{cm}^{-1}$ & SF-BSA + Propranolol NPs & $\mathrm{cm}^{-1}$ & SFm-NPs & $\mathrm{cm}^{-1}$ & $\begin{array}{c}\text { SFm + Propranolol } \\
\text { NPs }\end{array}$ \\
\hline & & 1652 & $\begin{array}{c}\text { Amide I } \\
\text { random coil }\end{array}$ & 1652 & $\begin{array}{l}\text { Amide I } \\
\text { random coil }\end{array}$ & 1631 & $\begin{array}{l}\text { Amide I } \\
\beta \text {-sheets }\end{array}$ \\
\hline 1645 & $\begin{array}{c}\text { BSA amide I } \\
\mathrm{C}=\mathrm{O} \text { stretching }\end{array}$ & 1644 & $\begin{array}{c}\text { BSA amide I } \\
\mathrm{C}=\mathrm{O} \text { stretching }\end{array}$ & 1549 & $\begin{array}{l}\text { Amide II } \\
\text { random coil }\end{array}$ & & \\
\hline 1634 & $\begin{array}{l}\text { Amide I } \\
\beta \text {-sheets }\end{array}$ & 1634 & $\begin{array}{l}\text { Amide I } \\
\beta \text {-sheets }\end{array}$ & & & 1466 & $\mathrm{CH}_{3}$ bending \\
\hline 1491 & $\begin{array}{l}\text { BSA amide II } \\
\mathrm{C}-\mathrm{N} \text { stretching } \\
\mathrm{N}-\mathrm{H} \text { bending }\end{array}$ & & & 1412 & $\mathrm{CH}_{3}$ bending & & \\
\hline 1487 & $\mathrm{CH}_{3}$ bending & 1467 & $\mathrm{CH}_{3}$ bending & 1384 & $\begin{array}{c}\mathrm{CH}_{2} \text { bending } \\
(\mathrm{AG})_{n}\end{array}$ & & \\
\hline 1403 & $\mathrm{CH}_{3}$ bending & & & 1264 & $\begin{array}{c}\text { Amide III } \\
\text { a-helices }\end{array}$ & 1274 & $\begin{array}{c}\text { Amide III } \\
\beta \text {-sheets }\end{array}$ \\
\hline 1363 & $\begin{array}{c}\mathrm{CH}_{2} \text { bending } \\
(\mathrm{AG})_{n}\end{array}$ & 1362 & $\begin{array}{l}\mathrm{CH}_{2} \text { bending } \\
(\mathrm{AG})_{n}\end{array}$ & 1226 & $\begin{array}{c}\text { Amide III } \\
\beta \text {-sheets }\end{array}$ & & \\
\hline 1262 & $\begin{array}{l}\text { Amide III } \\
\text { a-helices }\end{array}$ & 1261 & $\begin{array}{l}\text { Amide III } \\
\text { a-helices }\end{array}$ & & & & \\
\hline 1120 & $\begin{array}{c}\mathrm{CH}_{2} \mathrm{OH} \\
\text { polyphenols }\end{array}$ & 1120 & $\begin{array}{c}\mathrm{CH}_{2} \mathrm{OH} \\
\text { polyphenols }\end{array}$ & 1096 & $\begin{array}{l}\text { C-O stretching } \\
\text { C-C stretching } \\
\text { polyphenols or } \\
\text { tyrosine }\end{array}$ & & \\
\hline 1087 & $\begin{array}{l}\mathrm{C}-\mathrm{O} \text { stretching } \\
\mathrm{C}-\mathrm{C} \text { stretching }\end{array}$ & & & 1041 & $\begin{array}{l}\text { C-O stretching } \\
\mathrm{N}-\mathrm{C} \text { stretchingSer }\end{array}$ & & \\
\hline \multirow[t]{2}{*}{1041} & $\begin{array}{l}\text { C-O stretching } \\
\text { N-C stretching, } \\
\text { Ser }\end{array}$ & 1039 & $\begin{array}{l}\mathrm{C}-\mathrm{O} \text { stretching } \\
\mathrm{N}-\mathrm{C} \text { stretching, Ser }\end{array}$ & & & 952 & $\begin{array}{c}-\mathrm{CH}_{2} \\
(\mathrm{~A})_{n} \\
\beta \text {-sheets }\end{array}$ \\
\hline & & 952 & $\begin{array}{c}-\mathrm{CH}_{2} \\
(\mathrm{~A})_{n} \\
\beta \text {-sheets }\end{array}$ & 924 & $\begin{array}{c}-\mathrm{CH}_{2} \\
(\mathrm{~A})_{n} \\
\beta \text {-sheets }\end{array}$ & & \\
\hline 924 & $\begin{array}{c}-\mathrm{CH}_{2} \\
(\mathrm{~A})_{n} \\
\beta \text {-sheets }\end{array}$ & & & 595 & $\mathrm{Fe}-\mathrm{O}$ (magnetite) & 595 & $\mathrm{Fe}-\mathrm{O}$ (magnetite) \\
\hline
\end{tabular}

$(\mathrm{A})_{n}$, polyalanine; $(\mathrm{AG})_{n}$, polyalanine glycine [28].

\subsubsection{In Vitro Drug Release}

In Figure 8, the in vitro drug release profiles for the BSA- and magnetic-nanoparticles-modified silk fibroin nanoparticles are presented compared to the initial silk fibroin nanoparticles. Due to their higher encapsulation efficiency, the propranolol-encapsulated SF-BSA nanoparticles showed a higher drug release rate as compared to the propranolol-encapsulated silk fibroin nanoparticles [13]. In contrast, propranolol-encapsulated magnetic silk fibroin nanoparticles showed less release, which may be attributed to the strong electrostatic interactions of magnetite with the model drug. Another reason for the lower release could be the higher content of $\beta$-sheets in the propranolol-loaded silk fibroin magnetic nanoparticles causing a slow degradation of the silk fibroin and finally reducing the drug release rate [26]. 


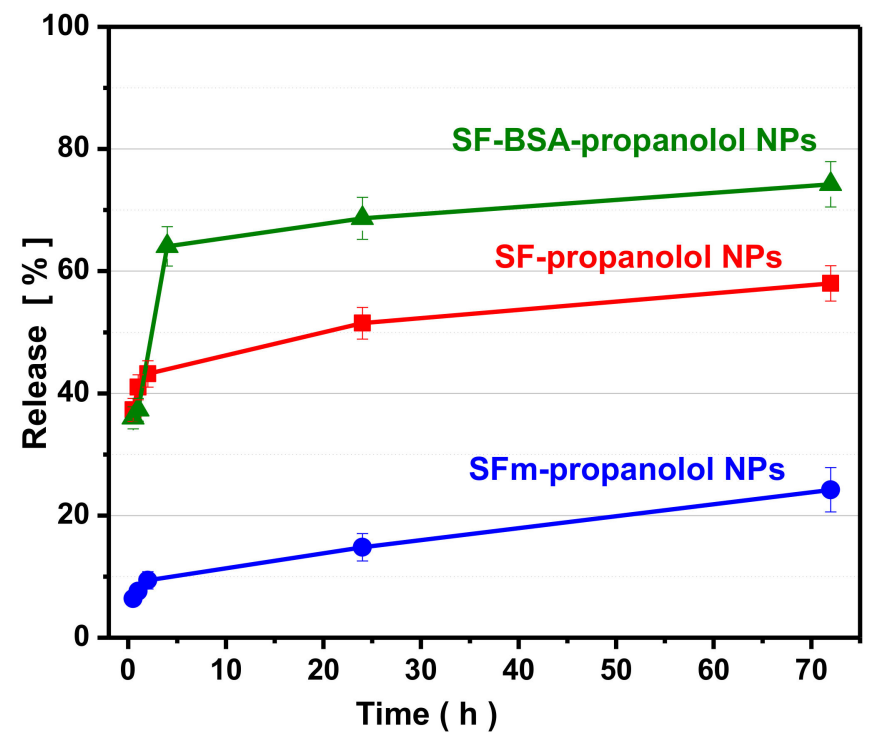

Figure 8. Drug release rate of fibroin nanoparticles, magnetic fibroin nanoparticles, and fibroin-bovine serum albumin nanoparticles encapsulated with propranolol.

\section{Conclusions}

Silk fibroin nanoparticles were prepared by an easy and safe-to-manipulate method that is amenable to a wide range of drugs and thus useful for silk-based drug delivery systems. Silk fibroin nanoparticles facilitated the entrapment of model drugs (curcumin, pramipexole, and propranolol) with different molecular weights and hydrophobicities and made drug release controllable. Silk fibroin particles loaded with propranolol exhibited a higher release percentage than those loaded with curcumin or pramipexole. Moreover, modified silk fibroin nanoparticles with magnetic nanoparticles as well as with bovine serum albumin were examined for the enhancement of the propranolol encapsulation and release from these nanoparticles. Magnetic silk fibroin nanoparticles increased the propranolol encapsulation efficiency. The silk fibroin-bovine serum albumin particles increased the encapsulation efficiency as well as the release rate of propranolol and were found to be a promising drug carrier.

Author Contributions: Olga Gianak and Eleni Deliyanni conceived and designed the experiments; Olga Gianak performed the experiments; Eleni Pavlidou performed the SEM measurements and analyzed the relative data; Charalampos Sarafidis performed the magnetic experiments and analyzed the relative data; Vassilis Karageorgiou performed the z-dynamic and dynamic light scattering measurements, analyzed the relative data, and contributed to the manuscript's writing; Eleni Deliyanni and Olga Gianak wrote the paper.

Conflicts of Interest: The authors declare no conflict of interest.

\section{References}

1. Tudora, M.R.; Zaharia, C.; Stancu, I.C.; Vasile, E.; Trusca, R.; Cincu, C. Natural silk fibroin micro-and nanoparticles with potential uses in drug delivery systems. UPB Sci. Bull. 2013, 75, 1454-2331.

2. Mottoghitalab, F.; Farokhi, M.; Shokrgozar, M.; Atyabi, M.A.F.; Hosseinkhani, H. Silk fibroin nanoparticle as a novel drug delivery system. J. Controll. Release 2015, 206, 161-176. [CrossRef] [PubMed]

3. Rahimnejad, M.; Mokhtarian, N.; Ghasemi, M. Production of protein nanoparticles for food and drug delivery system. Afr. J. Biotechnol. 2009, 8, 4738-4743.

4. Wang, X.; Yucel, T.; Lu, Q.; Hu, X.; Kaplan, D.L. Silk nanospheres and microspheres from silk/pva blend films for drug delivery. Biomaterials 2010, 31, 1025-1035. [CrossRef] [PubMed]

5. Numata, K.; Kaplan, D.L. Silk-based delivery systems of bioactive molecules. Adv. Drug Deliv. Rev. 2010, 62, 1497-1508. [CrossRef] [PubMed] 
6. Sieb, F.P. Silk nanoparticles-An emerging anticancer nanomedicine. AIMS Bioeng. 2017, 4, $239-258$. [CrossRef]

7. Subia, B.; Kundu, S.C. Drug loading and release on tumor cells using silk fibroin-albumin nanoparticles as carriers. Nanotechnology 2013, 24, 1-11. [CrossRef] [PubMed]

8. Karimi, M.; Bahrami, S.; Ravari, S.B.; Zangabad, P.S.; Mirshekari, H.; Bozorgomid, M.; Shahreza, S.; Sori, M.; Hamblin, M.R. Albumin nanostructures as advanced drug delivery systems. Expert Opin. Drug Deliv. 2016, 13, 1609-1623. [CrossRef] [PubMed]

9. Zhao, L.; Zhou, Y.; Gao, Y.; Ma, S.; Zhang, C.; Li, J.; Wang, D.; Li, X.; Li, C.; Liu, Y.; et al. Bovine serum albumin nanoparticles for delivery of tacrolimus to reduce its kidney uptake and functional nephrotoxicity. Int. J. Pharm. 2015, 10, 180-187. [CrossRef] [PubMed]

10. Tian, Y.; Jiang, X.; Chen, X.; Shao, Z.; Yang, W. Doxorubicin-Loaded Magnetic Silk Fibroin Nanoparticles for Targeted Therapy of Multidrug-Resistant Cancer. Adv. Mater 2014, 26, 7393-7398. [CrossRef] [PubMed]

11. Prijic, S.; Sersa, G. Magnetic nanoparticles as targeted delivery systems in oncology. Radiol. Oncol. 2011, 45, 1-16. [CrossRef] [PubMed]

12. Li, H.; Tian, J.; Wu, A.; Wang, J.; Ge, C.; Sun, Z. Self-assembly silk fibroin nanoparticles loaded with binary drug in the treatment of breast carcinoma. Int. J. Nanomed. 2016, 11, 4370-4380.

13. Gupta, V.; Aseh, A.; Rios, C.N.; Aggarwal, B.B.; Mathur, A.B. Fabrication and characterization of silk fibroin-derived curcumin nanoparticles for cancer therapy. Int. J. Nanomed. 2009, 4, 115-122. [CrossRef]

14. Kassas, R.A.; Wen, J.; Chen, A.E.M.; Kim, A.M.J.; Liu, S.S.M.; Yu, J. Transdermal delivery of propranolol hydrochloride through chitosan nanoparticles dispersed in mucoadhesive gel. Carbohydr. Polym. 2016, 153, 176-186. [CrossRef] [PubMed]

15. Leetjens, A.F.G.; Koester, J.; Fruh, B.; Shephard, D.T.S.; Barone, P.; Houben, J.J.G. The Effect of Pramipexole on Mood and Motivational Symptoms in Parkinson's Disease: A Meta-analysis of Placebo-Controlled Studies. Clin. Ther. 2009, 31, 89-98. [CrossRef] [PubMed]

16. Yu, Z.; Yu, M.; Zhang, Z.; Hong, G.; Xiong, Q. Bovine serum albumin nanoparticles as controlled release carrier for local drug delivery to the inner ear. Nanoscale Res. Lett. 2014, 9, 2-7. [CrossRef] [PubMed]

17. Rockwwod, D.N.; Preda, R.C.; Yucel, T.; Wang, X.; Lovett, M.L.; Kaplan, D.L. Materials fabrication from Bombyx mori silk fibroin. Nat. Protoc. 2011, 6, 1612-1631. [CrossRef] [PubMed]

18. Shi, P.; Goh, J.C.H. Release and cellular acceptance of multiple drugs loaded silk fibroin particles. Int. J. Pharm. 2011, 420, 282-289. [CrossRef] [PubMed]

19. Saroyan, H.; Giannakoudakis, D.; Sarafidis, C.; Lazaridis, N.; Deliyanni, E. Effective impregnation for the preparation of magnetic mesoporous carbon: Application to dye adsorption. J. Chem. Technol. Biotechnol. 2017, 92, 1899-1911. [CrossRef]

20. Leucuta, S.E.; Bodea, A. Optimization of propranolol hydrochloride sustained release pellets using a factorial design. Int. J. Pharm. 1997, 154, 49-57.

21. Li, M.; Lu, S.; Wu, Z.; Tan, K.; Minoura, N.; Kuga, S. Structure and properties of silk fibroin-poly-vinyl alcohol gel. Int. J. Biol. Macromol. 2002, 30, 89-94. [CrossRef]

22. Zhang, H.; Li, L.L.; Dai, F.Y.; Zhang, H.H.; Ni, B.; Zhou, W.; Yang, X.; Wu, Y.Z. Preparation and characterization of silk fibroin as a biomaterial with potential for drug delivery. J. Transl. Med. 2012, 10, 1-9. [CrossRef] [PubMed]

23. Zhao, Z.; Li, Y.; Xie, M.B. Silk fibroin-based nanoparticles for drug delivery. Int. J. Mol. Sci. 2015, 16, 4880-4903. [CrossRef] [PubMed]

24. Kamalha, E.; Zheng, Y.; Zeng, Y. Analysis of the secondary crystalline structure of regenerated Bombyx mori fibroin. Res. Rev. Biosci. 2013, 7, 76-83.

25. Huang, X.; Fan, S.; Altayp, A.I.; Zhang, Y.; Shao, H.; Hu, X.; Xie, M.; Xu, Y. Tunable Structures and Properties of Electrospun Regenerated Silk Fibroin Mats Annealed in Water Vapor at Different Times and Temperatures. J. Nanomater. 2014, 1-7. [CrossRef]

26. Reddy, M.K.D.; Sriniva, N.; Shanker, P. Formulation development and in vitro evaluation of pramipexole dihydrochloride monohydrate extended release matrix tablets. Int. J. Pharm. Sci. Res. 2014, 2, 2119-2133.

27. Xie, M.B.; Li, Y.; Zhao, Z.; Chen, A.Z.; Li, J.S.; Hu, J.Y.; Li, G.; Li, Z. Solubility enhancement of curcumin via supercritical $\mathrm{CO}_{2}$ based silk fibroin carrier. J. Supercrit. Fluids 2015, 103, 1-9. [CrossRef]

28. Kush, P.; Thakur, V.; Kumar, P. Formulation and in vitro evaluation of propranolol hydrochloride loaded polycaprolactone microspheres. Int. J. Pharm. Sci. Rev. Res. 2013, 20, 282-290. 
29. Audet, M.B.; Volrath, F.; Holland, C. Idnetification and classification of silks using infrared spectroscopy. J. Exp. Biol. 2015, 218, 3138-3149. [CrossRef] [PubMed]

30. Yu, S.; Yang, W.; Chen, S.; Chen, M.; Liu, Y.; Shao, Z.; Chen, X. Floxuridine-loaded silk fibroin nanospheres. RSC Adv. 2014, 4, 18171-18177. [CrossRef] 